\title{
M-CONSTANTS IN ORLICZ-LORENTZ SEQUENCE SPACES WITH APPLICATIONS TO FIXED POINT THEORY
}

\author{
YUNAN CUI*, PAWEŁ FORALEWSKI** AND HENRYK HUDZIK*** \\ *Department of Mathematics, Harbin University of Science and Technology \\ Harbin, 150080, China \\ E-mail: cuiya@hrbust.edu.cn \\ ${ }^{* *}$ Faculty of Mathematics and Computer Science, Adam Mickiewicz University \\ Umultowska 87, 61-614 Poznań, Poland \\ E-mail: katon@amu.edu.pl \\ *** Faculty of Economics and Information Technology, \\ The State University of Applied Sciences in P lock Nowe Trzepowo 55, 09-402 P lock, Poland \\ and Faculty of Mathematics and Computer Science, \\ Adam Mickiewicz University Umultowska 87, 61-614 Poznan, Poland \\ E-mail: hudzik@amu.edu.pl
}

\begin{abstract}
In this paper some estimates of $M$-constants in Orlicz-Lorentz sequence spaces for both, the Luxemburg and the Amemiya norms are given. Since degenerated Orlicz functions $\varphi$ and degenerated weighted sequences $\omega$ are also admitted, this investigations concern the most possible wide class of Orlicz-Lorentz sequence spaces. $M$-constants were defined in 1969 by E.A. Lifshits, and used in the study of lattice structures on Banach spaces, as well as in the fixed point theory, by a number of authors. In the last section of the paper an application of our results to the fixed point property is presented.

Key Words and Phrases: $M$-constants, $L$-constants, lattice structures on Banach spaces, Riesz angle, fixed point property, symmetric spaces, Orlicz-Lorentz spaces, Orlicz functions, Luxemburg norm, Amemiya norm.
\end{abstract}

2010 Mathematics Subject Classification: 46B20, 46B45, 46A45, 46B42, 46A80, 47H10.

\section{REFERENCES}

[1] Y.A. Abramovich, G.Y. Lozanovskiǐ, Certain numerical characteristics of KN-lineals, Matematiceskie Zametki, 14(1973), no. 5, 723-732 (in Russian).

[2] S.V. Astashkin, F.A. Sukochev, C.P. Wong, Distributionally concave symmetric spaces and uniqueness of symmetric structure, Adv. Mathematics, 232(2013), no. 1, 399-431.

[3] C. Bennett, R. Sharpley, Interpolation of Operators, Academic Press, Inc., New York, 1988.

[4] J.M. Borwein, B. Sims, Non-expansive mappings on Banach lattices and related topics, Houston J. Mathematics, 10(1984), no. 3, 339-356.

The first author is supported by NFC of Heilong Jiang Province A2015018.

The second and the third authors are supported partially by National Science Centre (NCN), Poland, Grant No. DEC - 2012/07/B/ST1/03360. 
[5] S. Chen, Geometry of Orlicz Spaces, Dissertationes Math. (Rozprawy Matematyczne), 356, Polish Academy of Sciences, Warsaw, 1996.

[6] S. Chen, H. Hudzik, On some convexities of Orlicz and Orlicz-Bochner spaces, Commentationes Math. Univ. Carol., 29(1988), no. 1, 13-29.

[7] P. Foralewski, On some geometric properties of generalized Orlicz-Lorentz sequence spaces, Indagationes Math. N. S., 24(2013), no. 2, 346-372.

[8] P. Foralewski, On some geometric properties of generalized Orlicz-Lorentz function spaces, Nonlinear Anal., 75(2012), no. 17, 6217-6236.

[9] P. Foralewski, H. Hudzik, R. Kaczmarek, M. Krbec, Miroslav, M. Wójtowicz, On the moduli and characteristic of monotonicity in Orlicz-Lorentz function spaces, J. Convex Anal., 20(2013), no. 4, 955-970.

[10] P. Foralewski, H. Hudzik, P. Kolwicz, Non-squareness properties of Orlicz-Lorentz sequence spaces, J. Funct. Anal., 264(2013), no. 2, 605-629.

[11] P. Foralewski, H. Hudzik, P. Kolwicz, Pawel, Non-squareness properties of Orlicz-Lorentz function spaces, J. Ineq. Appl. 2013, 2013:32, 25 pp.

[12] W. Gong, Z. Shi, Points of monotonicity in Orlicz-Lorentz function spaces, Nonlinear Anal., 73(2010), no. 5, 1300-1317.

[13] X. He, Y. Cui, H. Hudzik, The fixed point property of Orlicz sequence spaces equipped with the p-Amemiya norm, Fixed Point Theory Appl. 2013, 2013:340, 18 pp.

[14] H. Hudzik, A. Kamińska, M. Mastyło, Geometric properties of some Calderón-Lozanovskǐ space and Orlicz-Lorentz spaces, Houston J. Mathematics, 22,(1996), no. 3, 639-663.

[15] H. Hudzik, A. Kamińska, M. Mastyło, Monotonicity and rotundity properties in Banach lattices, The Rocky Mountain J. Mathematics, 30(2000), no. 3, 933-950.

[16] N.J. Kalton, Lattice Structures on Banach Spaces, Memoirs Amer. Math. Soc., 103(1993), no. 493.

[17] A. Kamińska, Some remarks on Orlicz-Lorentz spaces, Math. Nachrichten, 147(1990), 29-38.

[18] A. Kamińska, Extreme points in Orlicz-Lorentz spaces, Archiv der Mathematik, 55(1990), no. $2,173-180$

[19] A. Kamińska, Uniform convexity of generalized Lorentz spaces, Archiv der Mathematik, 56(1991), no. 2, 181-188.

[20] A. Kamińska, Y. Raynaud, Isomorphic copies in the lattice $E$ and its symmetrization $E^{(*)}$ with applications to Orlicz-Lorentz spaces, J. Funct. Anal., 257(2009), no. 1, 271-331.

[21] A. Kamińska, Y. Raynaud, New formulas for decreasing rearrangements and a class of OrliczLorentz spaces, Revista Matemática Complutense, 27(2014), no. 2, 587-621.

[22] A. Kamińska, K. Leśnik, Y. Raynaud, Dual spaces to Orlicz-Lorentz spaces, Studia Mathematica, 222(2014), no. 3, 229-261.

[23] A. Khamsi, W.M. Kozłowski, S. Chen, Some geometrical properties and fixed point theorems in Orlicz spaces, J. Math. Anal. Appl., 155(1991), no. 2, 393-412.

[24] W.A. Kirk, B. Sims, Handbook of Metric Fixed Point Theory, Kluwer Academic Publishers, Dordrecht-Boston-London, 2001

[25] S.G. Krein, J.I. Petunin, E.M. Semenov, Interpolation of Linear Operators, Translations of Mathematical Monographs, 54, American Math. Soc., Providence, 1982

[26] F.E. Levis, H.H. Cuenya, Gateaux differentiability in Orlicz-Lorentz spaces and applications, Math. Nachrichten, 280(2007), no. 11, 1282-1296.

[27] E.A. Lifshits, On the theory of partially ordered Banach spaces, Funktsional'nyi Analiz i ego Prilozheniya, 3(1969), no. 1, 91-92 (in Russian).

[28] J. Lindenstrauss, L. Tzafriri, Classical Banach Spaces I. Sequence spaces, Ergebnisse der Mathematik und ihrer Grenzgebiete, 92, Springer-Verlag, Berlin-New York, 1977.

[29] J. Lindenstrauss, L. Tzafriri, Classical Banach spaces II. Function spaces, Ergebnisse der Mathematik und ihrer Grenzgebiete, 97, Springer-Verlag, Berlin-New York, 1979.

[30] L. Maligranda, Indices and Interpolation, Dissertationes Math. (Rozprawy Matematyczne), 234, Polish Academy of Sciences, Warsaw, 1985.

[31] L. Maligranda, Orlicz Spaces and Interpolation, Seminars in Mathematics, 5, Universidade Estadual de Campinas, Campinas, SP, Brazil, 1989. 
[32] M. Mastyło, Lattice structures on some Banach spaces, Proc. Amer. Math. Soc., 140(2012), no. 4, 1413-1422.

[33] S.J. Montgomery-Smith, Comparison of Orlicz-Lorentz spaces, Studia Mathematica, 103(1992), no. 2, 161-189.

[34] S.J. Montgomery-Smith, Boyd indices of Orlicz-Lorentz spaces, in: Function Spaces, The Second Conference (Edwardsville, IL, 1994), (Jarosz, Krzysztof ed.) Marcel Dekker, Lecture Notes in Pure and Applied Math., 172, New York, 1995, 321-334.

[35] J. Musielak, Orlicz Spaces and Modular Spaces, Lecture Notes in Mathematics, 1034, SpringerVerlag, Berlin-Heidelberg-New York-Tokyo, 1983.

[36] B. Sims, Orthogonality and fixed points of nonexpansive maps, Workshop/Miniconference on Functional Analysis and Optimization (Canberra, 1988), Proc. Centre Math. Anal. Austral. Nat. Univ., Austral. Nat. Univ., Canberra, 20(1988), 178-186.

[37] C. Tsekrekos, Some applications of the L-constants and $M$-constants on Banach lattices, J. London Math. Soc., 18(1978), no. 2, 133-139.

[38] J. Wang, The Riesz angle of Orlicz function spaces, Commentationes Math., 40(2000), 209-223.

[39] W. Wnuk, Remarks concerning $M$-constants in Banach lattices, in: Proceedings of the Fourth International Symposium on Banach and Function Spaces, Kitakyushu, Japan 2012 (M. Kato, L. Maligranda, S. Tomonari Suzuki eds.), Yokohama Publishers, 2014, 259-273.

[40] Y. Yan, Riesz angles of Orlicz sequence spaces, Commentationes Math. Univ. Carol., 43(2002), no. 1, 133-147.

[41] Y. Yan, On some expressions for the Riesz angles of Orlicz sequence spaces, Siberian Math. J., 44(2003), no. 4, 739-747.

Received: July 18, 2016; Accepted: December 22, 2016. 